\title{
Characterization of DXZ4 conservation in primates implies important functional roles for CTCF binding, array expression and tandem repeat organization on the $\mathrm{X}$ chromosome
}

\author{
Christine R McLaughlin and Brian P Chadwick*
}

\begin{abstract}
Background: Comparative sequence analysis is a powerful means with which to identify functionally relevant noncoding DNA elements through conserved nucleotide sequence. The macrosatellite DXZ4 is a polymorphic, uninterrupted, tandem array of 3-kb repeat units located exclusively on the human $\mathrm{X}$ chromosome. While not obviously protein coding, its chromatin organization suggests differing roles for the array on the active and inactive $X$ chromosomes.

Results: In order to identify important elements within DXZ4, we explored preservation of DNA sequence and chromatin conformation of the macrosatellite in primates. We found that DXZ4 DNA sequence conservation beyond New World monkeys is limited to the promoter and CTCF binding site, although DXZ4 remains a GC-rich tandem array. Investigation of chromatin organization in macaques revealed that DXZ4 in males and on the active $X$ chromosome is packaged into heterochromatin, whereas on the inactive X, DXZ4 was euchromatic and bound by CTCF.
\end{abstract}

Conclusions: Collectively, these data suggest an important conserved role for DXZ4 on the X chromosome involving expression, CTCF binding and tandem organization.

\section{Background}

Macrosatellites are a type of variable number tandem repeat (VNTR) that primarily differ from other VNTRs by the size of the individual repeat unit (from 2 to $>12 \mathrm{~kb}$ ) and restriction of the array to one or two locations in the genome [1]. To date, at least eight different macrosatellite arrays have been described in the human genome [1-6], although several others remain largely unexplored [1].

Among the human macrosatellites, the best characterized is D4Z4, located at the subtelomeric regions of chromosomes 4q35 [6] and 10q26 [7,8]. D4Z4 consists of a tandem array of $3.3-\mathrm{kb}$ repeat units that can cover hundreds of kilobases at these chromosomal locations [6]. D4Z4 has been a major focus for research since a link

\footnotetext{
* Correspondence: chadwick@bio.fsu.edu

Department of Biological Science, Florida State University, 319 Stadium Drive, 3076 King Building, Tallahassee, FL 32306-4295, USA
}

was made between the array and facioscapulohumeral muscular dystrophy (FSHD) [6,9], an autosomal dominant disorder characterized by progressive atrophy of muscles in the face, shoulders and upper arms [10]. In almost all cases, FSHD onset is associated with contraction of the array at $4 \mathrm{q} 35$ to ten or fewer repeat units $[6,9]$. Contraction alone is not sufficient for disease onset, as pathogenesis is linked to a reduction in the number of D4Z4 repeat units on a defined haplotype termed 4qA161 [11]. Like other macrosatellites [1,2,5,12,13], D4Z4 is expressed [14]. Each D4Z4 monomer in the array contains an ORF [15] that encodes a double homeobox protein termed DUX4 [16]. Recent data indicate that transcripts originating from the most distal monomer are stabilized by a poly-adenylation signal located distal to the array [14,17], a feature that is only found on chromosomes with the 4qA161 haplotype [18]. These advances have focused attention on inappropriate

\section{() Biomed Central}


expression of DUX4 as the likely molecular basis of the disease.

Not all macrosatellites are obviously protein coding [5], which then begs the question of what role they fulfil in genome biology. One such example is the X-linked macrosatellite DXZ4. DXZ4 is a tandem array of 3-kb repeat units located at Xq23 [3]. While several short ORFs are present within a monomer, none have any homology to known proteins and they therefore may simply be a consequence of the high GC DNA sequence content reducing the incidence of stop codons. Identification of the ORF in D4Z4 was facilitated by the presence of the homeobox motif $[15,16]$, and further supported by conservation of the ORF through to rodents [19]. However, despite no clear ORF within a DXZ4 monomer, the array does adopt intriguing chromatin states in the context of X chromosome inactivation, which is the mammalian form of dosage compensation, a process that balances X-linked gene expression between the sexes by shutting down most gene expression from one of the two $\mathrm{X}$ chromosomes in females [20]. Gene silencing at the chosen inactive $X$ chromosome $(\mathrm{Xi})$ is achieved by repackaging the chromosome into facultative heterochromatin, including CpG island DNA hypermethylation [21,22], acquisition of covalent histone modifications associated with heterochromatin [23-26], and underrepresentation of euchromatic marks, including histone acetylation [27] and histone $\mathrm{H} 3$ dimethylated at lysine 4 (H3K4me2) [23]. Whereas most chromatin on the Xi adopts this new configuration, DXZ4 does not. CpG dinucleotides at DXZ4 are hypomethylated $[3,12]$ and DXZ4 nucleosomes are characterized by H3K4me2 [12,28] as well as several other euchromatic markers [29] and the array is bound by the multi-functional zinc finger protein CCCTC binding factor (CTCF) [12,29]. DXZ4 at the Xi is readily detected as a signal of H3K4me2 within the hypo-H3K4me2 territory of the Xi at interphase $[28,29]$, and as a distinct signal midway down the long arm of the chromosome at metaphase [28]. Facultative heterochromatin of the Xi is not a homogenous mass of likechromatin, but is instead composed of at least two different types that occupy reproducible alternating bands along the Xi $[30,31]$. DXZ4 resides at the interface of two such bands [28], and therefore may have some role in organization of the Xi involving CTCF. In sharp contrast, DXZ4 CpG dinucleotides on the active X chromosome (Xa) and in males are hypermethylated $[3,12]$ and nucleosomes are packaged into constitutive heterochromatin characterized by histone $\mathrm{H} 3$ lysine- 9 trimethylation (H3K9me3) [12] and binding of heterochromatin protein 1 gamma (HP1 $\gamma)$ [32]. Therefore, human DXZ4 adopts alternative chromatin states on the $\mathrm{Xa}$ and $\mathrm{Xi}$ that differ from the surrounding chromosome.
Intriguingly, analysis of D4Z4 chromatin organization in FSHD patients revealed that the contracted allele adopted a more euchromatic organization [32,33] involving CTCF binding [34], hence resembling DXZ4 on the $\mathrm{Xi}$ [35]. Therefore, these findings highlight how investigating the biology of macrosatellites in general can provide insight into the function of other macrosatellites such as D4Z4 in the context of FSHD.

In order to further understand DXZ4, we sought to identify conserved DNA sequence and chromatin organization for the array by investigating the macrosatellite in other primates, and report our findings here.

\section{Results}

DXZ4 is a conserved X-linked macrosatellite repeat in Old World and New World monkeys

Previously, DXZ4 has been assigned to the X chromosome by fluorescence in situ hybridization (FISH) in gorilla, chimpanzee and orangutan [36], but nothing further is known about conservation of DXZ4 outside of the great apes. To determine if DXZ4 is sufficiently conserved in Old World monkeys, human probes for DXZ4 and the gene $70 \mathrm{~kb}$ proximal to the human array (PLS3) were hybridized to male and female rhesus macaque (Macaca mulatta) metaphase chromosomes. An intense signal for DXZ4 was detected on a single chromosome in the male sample and on two chromosomes in the female samples (Figure 1a). In both cases, the DXZ4 signal partially overlapped with the PLS3 signal. Collectively, these data indicate that DXZ4 in macaque is $\mathrm{X}$-linked and its chromosomal location is conserved adjacent to PLS3.

Next we sought to determine if DXZ4 is a polymorphic tandem array in Old World monkeys and if DXZ4 sequence conservation was sufficiently high enough to detect DXZ4 in New World monkeys using a human DXZ4 probe. Agarose embedded genomic DNA from a human sample as a hybridization control, one female gorilla, two male macaques, two female macaques and a male and female New World monkey were cut with $\mathrm{XbaI}$ and fragments separated by pulsed field gel electrophoresis. Given that there are no recognition sites for XbaI in human DXZ4, genomic DNA will be cut at the first available sites proximal and distal to DXZ4, essentially excising the array intact. Because the copy number of DXZ4 monomers in humans varies between individuals, hybridizing DNA fragments are polymorphic [3]. A Southern blot of the gel was hybridized with the human probe before washing to high stringency $\left(0.2 \times \mathrm{SSC}, 0.1 \% \mathrm{SDS}\right.$ at $\left.60^{\circ} \mathrm{C}\right)$. A single hybridizing band was detected in all male samples, and two bands in the females, including the New World monkeys (Figure 1b). Three conclusions can be drawn from this result. First, DXZ4 sequence is conserved in 


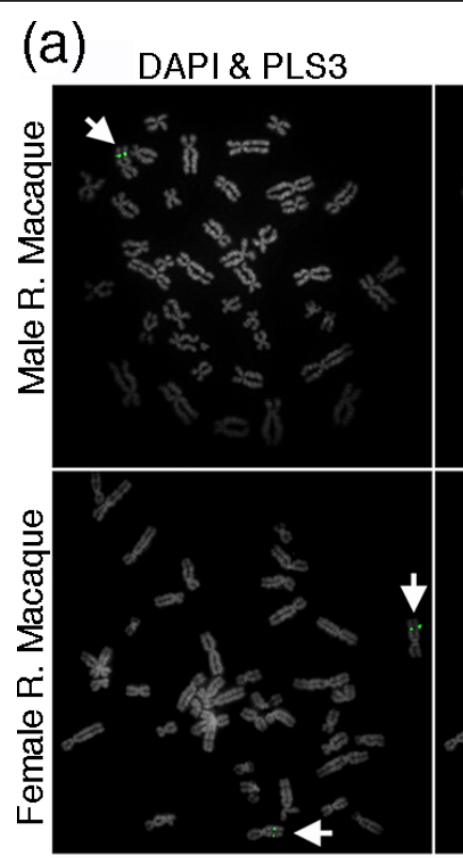

\section{DAPI \& DXZ4 DAPI, PLS3 \& DXZ4}

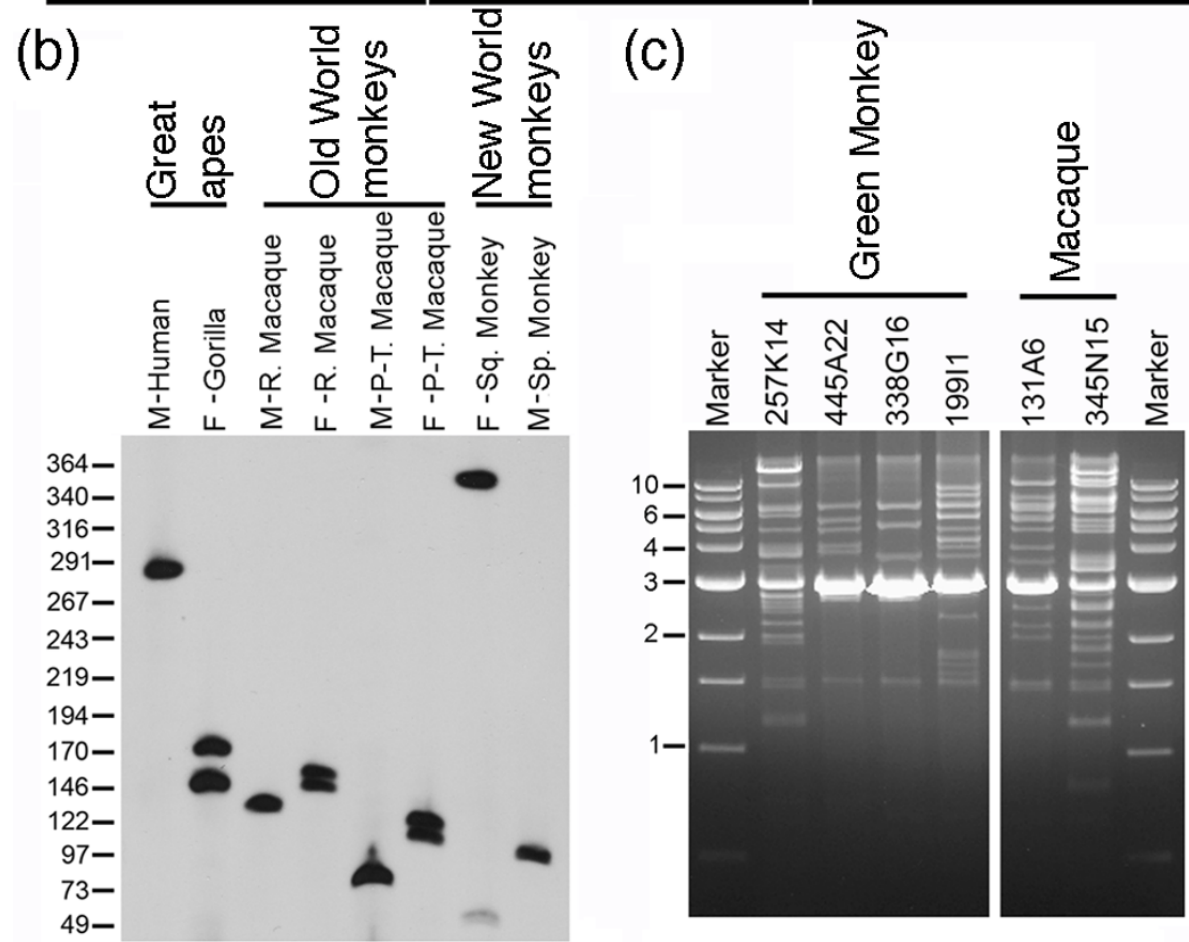

Figure 1 Mapping and tandem arrangement of DXZ4 in primates. (a) Direct-labeled fluorescence in situ hybridization (FISH) of human DXZ4 BAC clone (2272M5; red) and human PLS3 BAC clone (268A15; green) to male and female rhesus macaque metaphase chromosomes. White arrows point to the hybridizing $X$ chromosome. Metaphase chromosomes were counterstained with DAPI, and converted to gray-scale to assist in visualizing the FISH signals. (b) Southern blot of Xbal digested primate genomic DNA separated by pulsed field gel electrophoresis, hybridized with a human digoxigenin-labeled DXZ4 probe. Primates and group are listed along the top and gender indicated by M (male) or F (female), including rhesus macaque (R. Macaque), pig-tailed macaque (P-T. Macaque), common squirrel monkey (Sq. Monkey) and black-handed spider monkey (Sp. Monkey). Size in kilobases is given to the left. (c) Ethidium bromide stained $0.9 \%$ agarose gel showing green monkey and macaque BAC DNA digested with the restriction endonuclease Hindlll and separated by gel electrophoresis. The sizes of the molecular weight marker are given to the left in kilobases. 
primates at least as far as New World monkeys. Second, given the single male band and two female bands, this further supports X-linkage for DXZ4 in primates. Finally, the range of hybridizing fragments (approximately 50 to $350 \mathrm{~kb}$ ) indicates that DXZ4 is a VNTR in the great apes, Old World monkeys and New World monkeys.

Comparison of the human DXZ4 DNA sequence [GenBank: HQ659140] against the rhesus macaque genome sequence (rheMac2), did not identify a VNTR, but instead identified various broken matches over short intervals and the presence of a large gap in the genome sequence, indicating that like many tandem repeat DNAs in the various early releases of the human genome, the sequence of DXZ4 is poorly assembled in the current build of the macaque genome. In order to further characterize DXZ4 in Old World monkeys, we compared the human DXZ4 sequence to entries in the public databases using BLAST. Several green monkey (Cercopithecus aethiops) and rhesus macaque BAC clones were identified with matches to DXZ4 that were then ordered and obtained. Human DXZ4 is cut once per monomer with HindIII [3]. Digestion of the Old World monkey BACs with HindIII revealed an over representation of a 3-kb fragment in the BAC clones (Figure 1c), supporting the presence of multiple DXZ4 sequences in the clones. The $3-\mathrm{kb}$ HindIII band was excised from the gel and cloned before sequencing.

Comparison of either the complete 2,950 bp green monkey sequence [GenBank: HQ906499] or the complete 2,922 bp rhesus macaque sequence [GenBank: HQ906498] against human DXZ4 (2,994 bp) using ClustalW2 [37] revealed $87 \%$ nucleotide identity in both cases (Table 1 ). This is lower than the observed sequence divergence of $94 \%$ between the human and macaque X chromosomes [38]. Directly comparing the two Old World monkey DXZ4 sequences revealed 95\% sequence identity.

Using the public databases, additional complete monomer sequences were extracted for chimpanzee, gorilla, white-cheeked gibbon (Hylobates leucogenys) and a New World monkey (Callithrix jacchus or the common marmoset) (Table 1). These sequences were then compared to human DXZ4. As expected, the percent nucleotide identity to human DXZ4 was highest within the great apes, lower in the lesser apes, lower still in Old World monkeys and lowest in New World monkeys. In all cases, the monomers were similar in size, GC content and the number of CpG dinucleotides. The DNA sequence of human DXZ4 is unique to its location on the X chromosome, with the exception of three internal polymorphic microsatellite sequences [3]; [GGGCC]n, [CT]n and [TAAA]n (Figure 2). In humans, copy number of these sequences accounts for most variation in DXZ4 between adjacent monomers (Tremblay DC et al., in preparation). In chimpanzee, all three of the microsatellite sequences are conserved. However, in gorilla the [TAAA] repeat is replaced by a [CAAAAAA] repeat, and by an A-rich sequence in gibbon (Figure 2). Both Old World monkey DXZ4 monomers contained 3 copies of [GGGCC], with 19 [CT] in rhesus macaque and $25[\mathrm{CT}]$ in the green monkey that were interrupted by two [GT]. Neither Old World monkey contained the [TAAA] repeat. Instead, the sequence was replaced with a 22-bp poly-A stretch in rhesus macaque, and 4 copies of [GAAA] in the green monkey (Figure 2). In the New World monkey, the [GGGCC] repeat is replaced by a [TGGGG] repeat. The [CT] repeat is still present, but immediately adjacent to this microsatellite is a new dinucleotide [CA] repeat. The [TAAA] repeat is also replaced by a [CCAAA] repeat. Collectively, these data indicate that the [TAAA] repeat is the least conserved internal repeat in primate DXZ4, but that all primates retain simple repeat DNA of similar base composition at these regions (Figure 2).

Tandem repeat organization is retained in lemurs, but sequence conservation is restricted to the promoter and CTCF binding region of human DXZ4 in distantly related primates

The DNA sequence of human DXZ4 was used to identify homologous sequences from other primate entries in the public databases. Several matches were made with sequences from Sumatran orangutan (Pongo pygmaeus abelii), gray mouse lemur (Microcebus murinus), ringtailed lemur (Lemur catta), small-eared galago (Otolemur

Table 1 Comparison between human DXZ4 and monomer sequences identified in great ape, lesser ape, Old World monkey and New World monkey

\begin{tabular}{|c|c|c|c|c|}
\hline Primate & Monomer size (bp) & Percent identity to human & GC content & CpG \\
\hline Human & 2,994 & - & $62 \%$ & 186 \\
\hline Chimpanzee & 2,996 & $97 \%$ & $62 \%$ & 176 \\
\hline Gorilla & 2,954 & $95 \%$ & $62 \%$ & 165 \\
\hline Gibbon & 2,896 & $90 \%$ & $63 \%$ & 166 \\
\hline Macaque & 2,922 & $87 \%$ & $62 \%$ & 161 \\
\hline Green monkey & 2,950 & $87 \%$ & $61 \%$ & 154 \\
\hline Marmoset & 3,039 & $77 \%$ & $65 \%$ & 192 \\
\hline
\end{tabular}




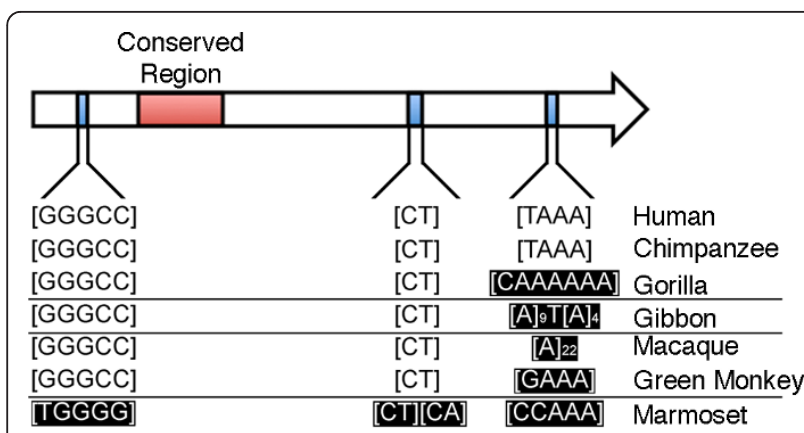

Figure 2 Conservation of simple repeat sequences at DXZ4 DXZ4 is represented by a right facing 3-kb monomer. Annotated on this monomer is the region of DXZ4 that is conserved in all primates examined (red box) as well as the location of the three internal simple repeat sequences (blue boxes), which account for all repetitive DNA in DXZ4. The sequence of the simple repeats in human is given immediately below the schematic map and beneath this the nucleotide composition in various other primates is given. Sequences that diverge from the human sequence are highlighted as white writing on a black background. The horizontal lines divide great apes from the lesser apes, Old World monkeys and New World monkeys.

garnettii) and the Philippine tarsier (Tarsius syrichta). However, for the more distantly related primates (lemurs, galagos and tarsiers), sequence matches were limited to a 402-bp interval of DXZ4 corresponding to the site where the epigenetic organizer protein CTCF is bound and bidirectional promoter activity has been assigned [12] (Figure 3a). Outside of this region, limited sequence homology can be detected, with the exception of an additional 326 bp that shares $69 \%$ broken homology with ringtailed lemurs (data not shown). Using the most conserved 402-bp sequence from all primates, a phylogenetic tree was assembled (Figure 3b). The resulting cladogram is similar to one generated through investigation of mobile element distribution in primates [39]. Interestingly, sequence identity for this interval was higher than across the whole monomer through New World monkeys (compare Table 1 to Figure $3 \mathrm{~b}$ ), supporting this region as the most conserved part of DXZ4. It is important to note that the available sequence data for DXZ4 in many of the primates shown is limited. As more sequences are released into the public domain, it is conceivable that the phylogenetic relationship of at least the distantly related primates may change. Nevertheless, it is clear that sequence conservation across a single DXZ4 monomer breaks down outside of the New World monkey branch of the primate tree.

The ring-tailed lemur sequence was derived from a BAC clone (LB2-162N9) [GenBank: AC133072] within which a $170-\mathrm{kb}$ continuous sequence is assembled. The first $70 \mathrm{~kb}$ of this sequence aligns with the 402-bp human DXZ4 sequence 15 times (data not shown), indicating a locally repetitive nature for this interval. Of the remaining $100 \mathrm{~kb}$,

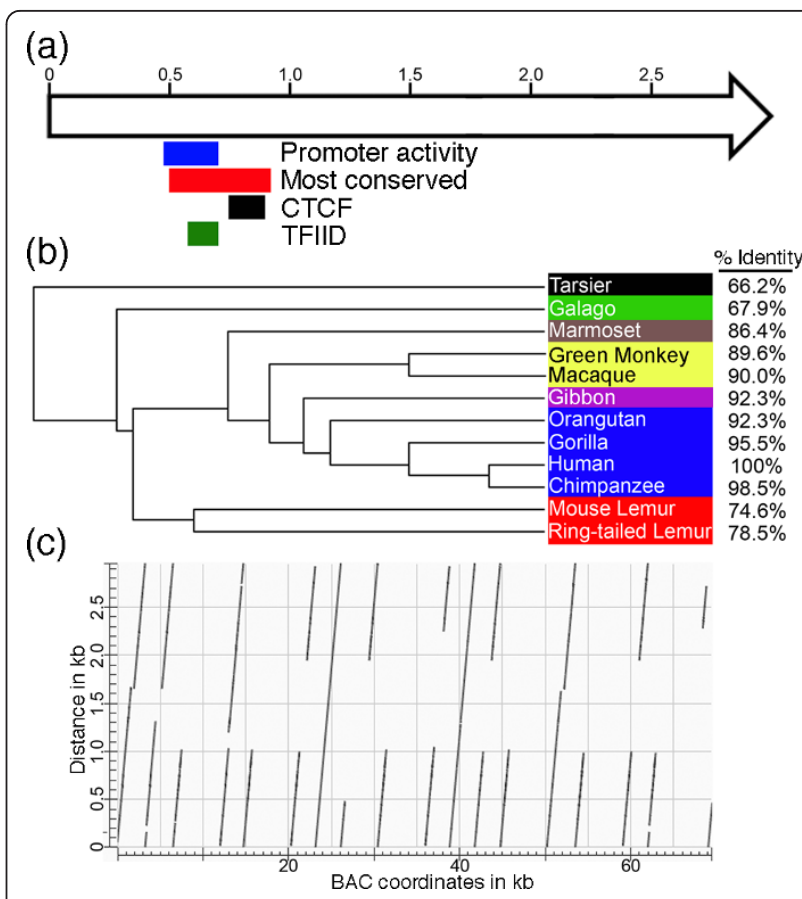

Figure 3 DNA sequence conservation of DXZ4. (a) Schematic map of a single human 3-kb DXZ4 monomer represented by an open arrow. Distance in kilobases is given along the top. Underneath, the colored bars indicate the regions of DXZ4 corresponding to the defined promoter, CTCF and TFIID binding sites [12] and the 402-bp interval of DXZ4 that is most conserved in primates. (b) Phylogenetic tree showing inferred evolutionary relationship of DXZ4 in primates. Sequence alignments were made against a 402 nucleotide region of human DXZ4 that is most conserved in all primates (nucleotides 517 to 918 of accession number [GenBank:HQ659140]). Percentage nucleotide identity is indicated to the far right. Primates are color-coded as follows: blue, great apes; pink, lesser apes; yellow, Old World monkeys; brown, New World monkeys; red, lemurs; green, galago; black, tarsier. The tree image was generated using MUSCLE version 3.8 [45]. (c) Predicted higher-order organization of the ring-tailed lemur DXZ4 sequence as revealed by dot-plot analysis. A single 3-kb DNA sequence is on the $y$-axis, whereas approximately $70 \mathrm{~kb}$ of $\mathrm{BAC}$ clone LB2-162N9 is given on the $x$-axis. The dot-plot was generated using the default settings for NCBI Blast2, and the output image labeled in Adobe Photoshop CS2.

multiple extensive single copy matches are made with unique DNA sequences found distal to human DXZ4, indicating that the BAC clone likely contains part of the orthologous ring-tailed lemur DXZ4 array. Comparison of the 170-kb BAC sequence against itself confirmed the presence of a more extensive tandem repeat in the first $70 \mathrm{~kb}$ than just the 402-bp sequence. Alignment of a 3-kb sequence from within this interval against the $70-\mathrm{kb}$ sequence clearly demonstrates that the DNA has characteristics of a tandem repeat (Figure 3c). However, unlike human DXZ4 (Tremblay DC et al., in preparation), the sequence is not a perfect tandem array of uninterrupted monomers of a uniform size, but consistent with other 
primates (Table 1), the 3-kb sequence is characterized by high GC content (62\%).

\section{Conserved hypo-methylation of $\mathrm{CpG}$ residues on the macaque $\mathrm{Xi}$}

In humans, DXZ4 on the male $\mathrm{X}$ and $\mathrm{Xa}$ in females is characterized by $\mathrm{CpG}$ methylation, whereas $\mathrm{CpGs}$ on the $\mathrm{Xi}$ are hypomethylated $[3,12]$. To determine if this was conserved at the macaque $\mathrm{Xi}$, we prepared bisulfite modified genomic DNA from a male and female rhesus macaque and pig-tailed macaque primary fibroblast culture, and then proceeded to PCR amplify a 621-bp fragment spanning the region corresponding to the human promoter and CTCF binding site (Figure 4a). PCR products were TA cloned and at least 20 independent clones were sequenced. Consistent with humans, male DXZ4 is extensively methylated (Figure 4b, left panels). In contrast, female DXZ4 is hypomethylated in a little over half of all clones (Figure 4b, right panels). The logical interpretation of these data is that, like humans,
DXZ4 in macaque is hypomethylated on the Xi. Notably, a single CpG residue is unmethylated in all male and female pig-tailed macaque clones (Figure 4b, bottom panels). The significance of this is unclear, although it does contribute to the lower overall percentage methylation observed in this species.

\section{Conserved H3K4me2 banding on the macaque $\mathrm{Xi}$}

At metaphase, H3K4me2 is largely absent from the human $\mathrm{Xi}$, with the exception of the tip of Xp including the pseudoautosomal region and DXZ4 midway down Xq $[28,29]$. Comparing the human H3K4me2 banding pattern to heterochromatin features of the $\mathrm{Xi}$ indicates that the euchromatic mark resides at the distal edge of a major band of histone $\mathrm{H} 3$ lysine-27 trimethylation (H3K27me3) and macroH2A [28,30,31]. We therefore sought to determine if the banding pattern of H3K4me2 on the macaque $\mathrm{Xi}$ resembled that seen in humans. In female rhesus macaque, a single chromosome was consistently marked by bands of H3K27me3 and hypo-

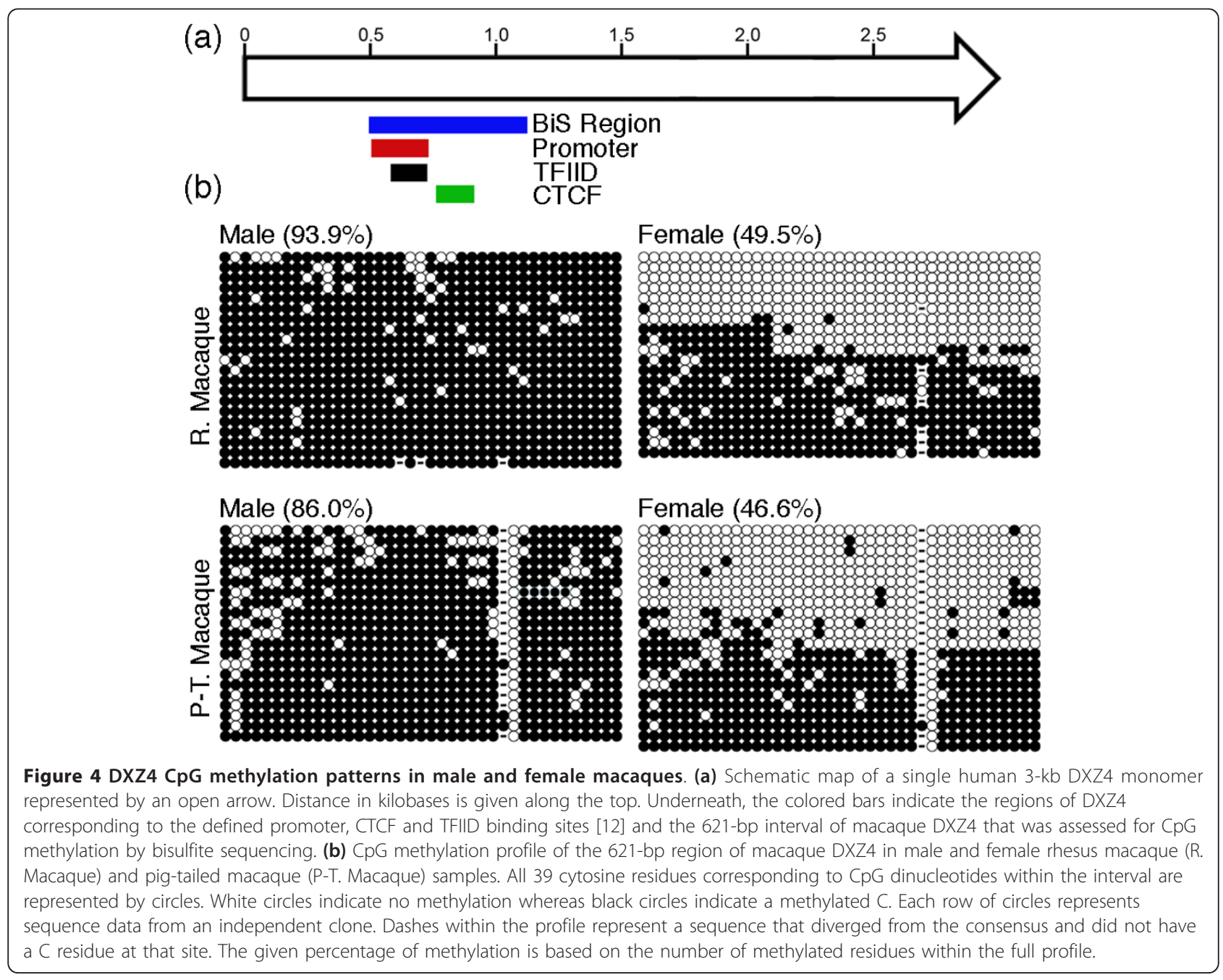


H3K4me2 (an example is shown in Figure 5a), features typically characteristic of the Xi [28,30,31]. Close examination of the chromosome revealed an intense H3K4me2 signal midway down the long arm that resided at the distal edge of a major band of $\mathrm{H} 3 \mathrm{~K} 27 \mathrm{me} 3$ (Figure 5b), and in the vicinity of DXZ4 and PLS3 FISH signals (Figure 1a). Additional H3K4me2 signals were observed at the tip of the p-arm, consistent with the pseudoautosomal region, and at the distal edge of a second q-arm band of H3K27me3. H3K4me2 band locations were reproducible on the macaque $\mathrm{Xi}$, consisting predominantly of the major q-arm signal and other weaker bands on the $\mathrm{p}$ and $\mathrm{q}$ arms (Figure $5 \mathrm{c}$ ). A similar pattern is observed at the human $\mathrm{Xi}$ (compare to Figure $5 \mathrm{~d}$ ), indicating conserved chromatin structure at these regions.

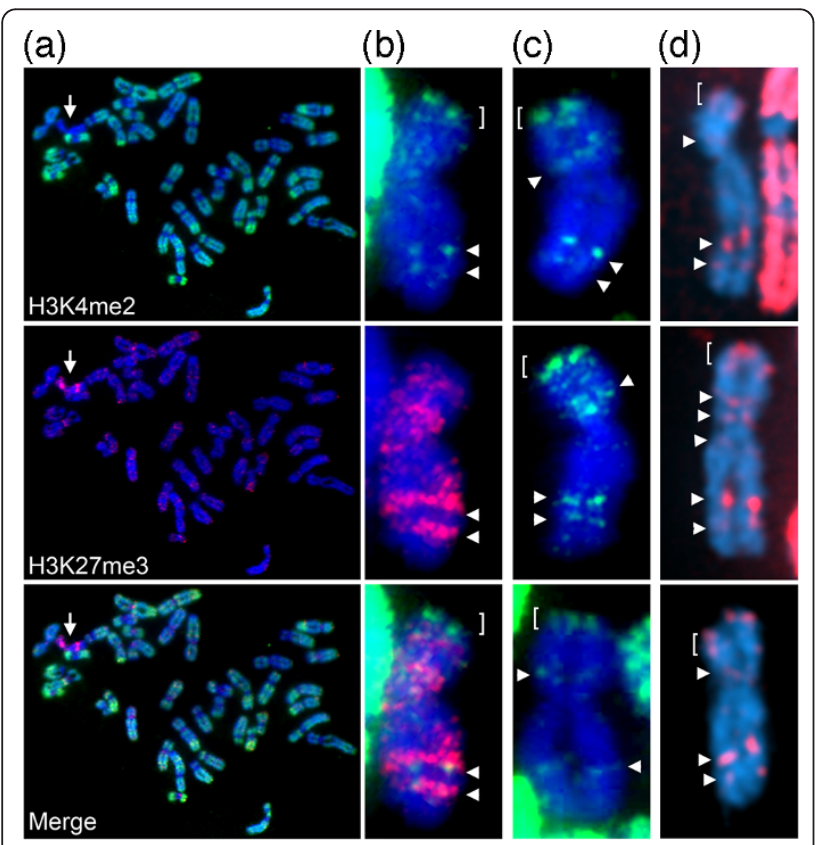

Figure 5 H3K4me2 distribution on the macaque metaphase $\mathrm{Xi}$. (a) Female rhesus macaque metaphase chromosomes (blue) showing the distribution of H3K4me2 (green) and H3K27me3 (red) by indirect immunofluorescence. The location of the $X_{i}$ is indicated by the white arrow. (b) Female rhesus macaque metaphase Xi (blue) showing the distribution of H3K4me2 (green) and H3K27me3 (red) by indirect immunofluorescence. The tip of the p-arm including the pseudoautosomal region is indicated by the white square bracket. Additional H3K4me2 bands on the Xi are indicated by the white arrowheads. (c) Further examples of H3K4me2 band distribution on the $\mathrm{Xi}$ to demonstrate reproducibility of the pattern. Signals indicated as for (b). (d) Human female telomerase immortalized retinal pigment epithelial cell (hTERT-RPE1) Xi showing the location of H3K4me2 (red) signals, as indicated by the white arrowheads and white bracket.
Euchromatic markers are largely absent from the macaque $\mathrm{Xi}$ with the exception of a discrete signal within the interphase territory of the chromosome

At interphase, euchromatic markers are absent from the human $\mathrm{Xi}$ with the notable exception of a signal within the territory of the $\mathrm{Xi}[23,28,29]$ that is inseparable from DXZ4 [29]. Two covalent modifications of histone $\mathrm{H} 3$ that are associated with transcription, H3 lysine-4 trimethylation (H3K4me3) and $\mathrm{H} 3$ lysine-36 dimethylation (H3K36me2), as well as lysine acetylation (a general euchromatin modification) were examined in rhesus macaque. The three modifications in male cells showed a general nuclear distribution that was absent from the nucleoli and little to no overlap with territories of facultative heterochromatin defined by H3K27me3 (Figure 6, left panels). In female cells, the nuclear distribution was like that of males with the additional observation that the $\mathrm{Xi}$ (defined by H3K27me3) lacked signals for the euchromatin markers (Figure 6, right panels). Both H3K4me3 and H3K36me2 showed the presence of a signal within the territory of the $\mathrm{Xi}$, a feature that was occasionally seen for the pan-acetyl lysine antibody.

\section{Xi DXZ4 in macaque is characterized by $\mathrm{H} 3 \mathrm{~K} 4 \mathrm{me} 2$ and CTCF}

In humans, $\mathrm{H} 3 \mathrm{~K} 4 \mathrm{me} 2$ is a feature of DXZ4 chromatin on the $\mathrm{Xi}$ as is the association of the epigenetic organizer and insulator protein CTCF $[12,29]$. We sought to determine if CTCF was a feature of macaque DXZ4 Xi. We used H3K4me2 to define the territory of the $\mathrm{Xi}$ and location of the DXZ4 dot in female macaque nuclei, and compared this to the nuclear distribution of CTCF. As in humans, CTCF was largely absent from the $\mathrm{Xi}$ with the exception of a signal that overlapped with the H3K4me2 'dot' (Figure 7a).

To confirm DXZ4 as the site of H3K4me2 and CTCF, chromatin immunoprecipitation was performed on chromatin prepared from male and female rhesus macaque and pig-tailed macaque, along with the heterochromatin marker H3K9me3. Both male and female samples showed the presence of $\mathrm{H} 3 \mathrm{~K} 9 \mathrm{me} 3$, whereas H3K4me2 and CTCF were readily detected in the female samples from both species of macaque (Figure 7b). The logical interpretation of these data is that DXZ4 in males and on the Xa is characterized by constitutive heterochromatin, whereas DXZ4 on the $\mathrm{Xi}$ is characterized, at least in part, by a euchromatic conformation bound by CTCF, consistent with that seen for human DXZ4 [12].

\section{Macaque DXZ4 is transcribed}

In humans, DXZ4 is expressed [12]. Transcripts are readily detected from DXZ4 packaged as constitutive 


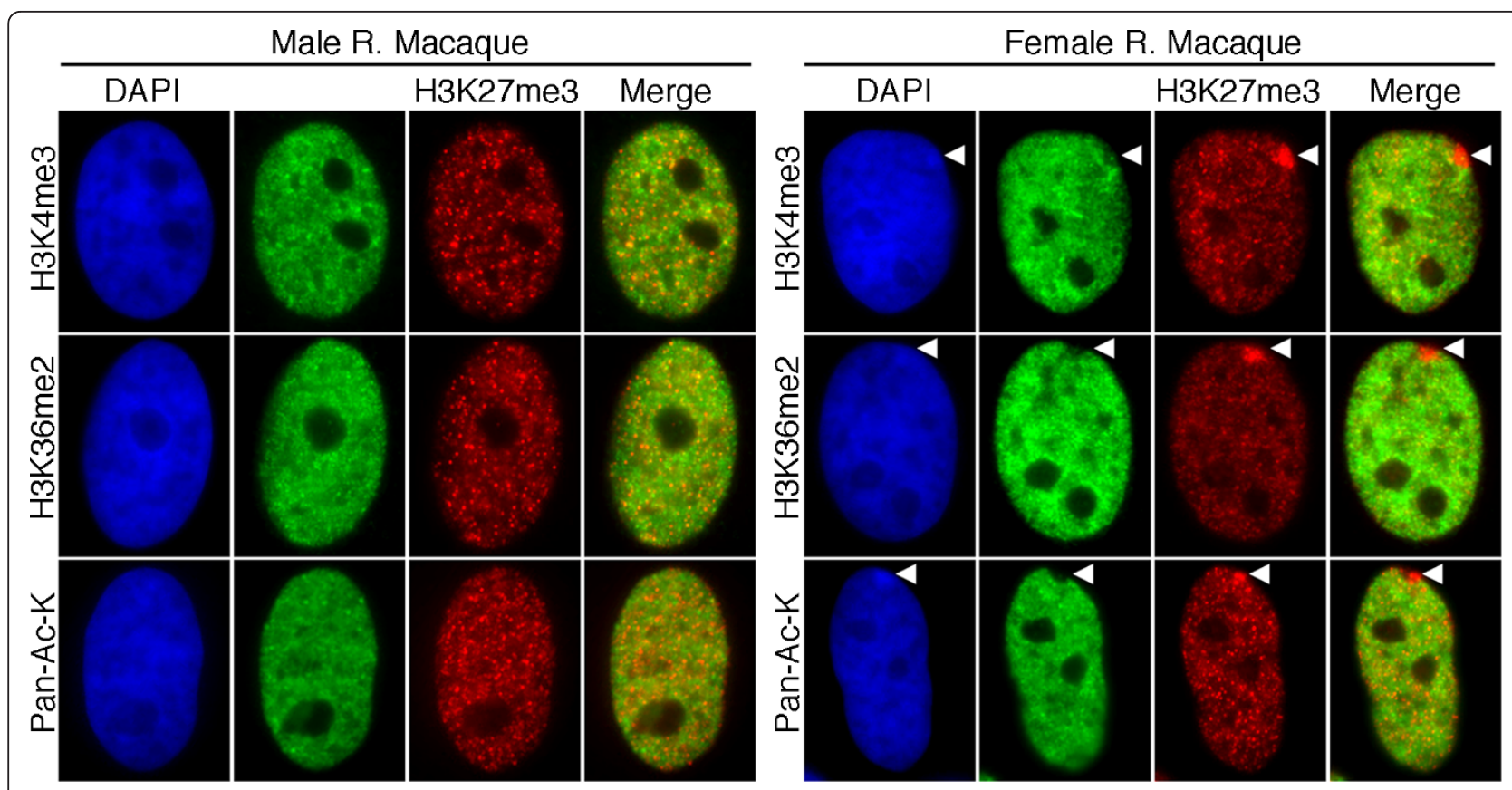

Figure 6 Distribution of euchromatic chromatin marks relative to the macaque Xi at interphase. Typical examples of male and female rhesus macaque (R. Macaque) interphase nuclei showing the distribution of H3K27me3 (red), H3K4me3 (green), H3K36me2 (green) and acetylated lysine (Pan-Ac-K, green) as determined by indirect immunofluorescence. Nuclei are counterstained with DAPI (blue). White arrowheads in the female images indicate the location of the Xi.

heterochromatin in male samples, as well as from the $\mathrm{Xi}$ in females, although whether transcripts originate from the heterochromatic or euchromatic portion of the $\mathrm{Xi}$ array is unclear. Transcript levels are highly variable between cells. In females DXZ4 can be detected by RNA $\mathrm{FISH}$ originating from either the $\mathrm{Xa}$ or $\mathrm{Xi}$ only, or from both chromosomes simultaneously (Tremblay DC et al., in preparation). We isolated total RNA from male and female rhesus macaque fibroblasts, prepared cDNA and performed PCR with primer sets corresponding to three regions of macaque DXZ4 (Figure 8a). DXZ4 was readily detected in the male and female samples. In order to determine if DXZ4 expression levels varied significantly between males and females, quantitative RT-PCR (qRTPCR) was performed on the cDNA. Levels of DXZ4 were comparable in the male and female samples analyzed (Figure 8b). In humans, most DXZ4 transcript originates from the sense strand, whereas low levels of anti-sense transcript can be detected originating form the Xi [12]. In order to assess DXZ4 transcript origins, strand-specific cDNA was prepared. In both the male and female samples, transcript could only be detected originating from the sense strand (Figure 8c), contrasting with humans. However, it is important to note that amounts of anti-sense DXZ4 transcript in humans are low, and DXZ4 transcription levels vary significantly between human samples (Tremblay DC et. al., in preparation). Therefore, in order to conclude that macaque does not generate anti-sense transcript, this analysis would need to be extended to numerous other independent macaque samples and cell types. Next we performed RNA FISH with a DXZ4 and XIST (X inactive specific transcript) probe to determine if expression is only from the $\mathrm{Xa}$, or if it can also originate from the Xi. Male macaque samples showed a clear signal for DXZ4 transcripts and no XIST RNA (Figure 8d, top panels). Female macaque samples also showed clear expression of DXZ4 (Figure 8d, bottom panels), although like males almost all transcripts originated from the Xa with only $6 \%$ of female rhesus macaque showing expression from the $\mathrm{Xi}$ and none in female pig-tailed macaque (Figure 8e). To ensure that this did not reflect an inability to detect DXZ4 transcripts at the $\mathrm{Xi}$, a control experiment was performed using a probe to the pseudoautosomal MIC2 gene. MIC2 transcripts were detected at the $\mathrm{X}$ and $\mathrm{Y}$ in males and $\mathrm{Xa}$ and $\mathrm{Xi}$ in females (Figure $8 \mathrm{f}$ ). Therefore, while expression of DXZ4 is conserved in macaque, the relevance of the antisense transcript is unclear.

\section{Discussion}

To identify functionally important features of DXZ4, we investigated chromatin structure of the array in the Old World monkey macaque, expression and retention of tandem repeat organization, and primary DNA sequence conservation in a variety of closely and distantly related members of the primate lineage. 


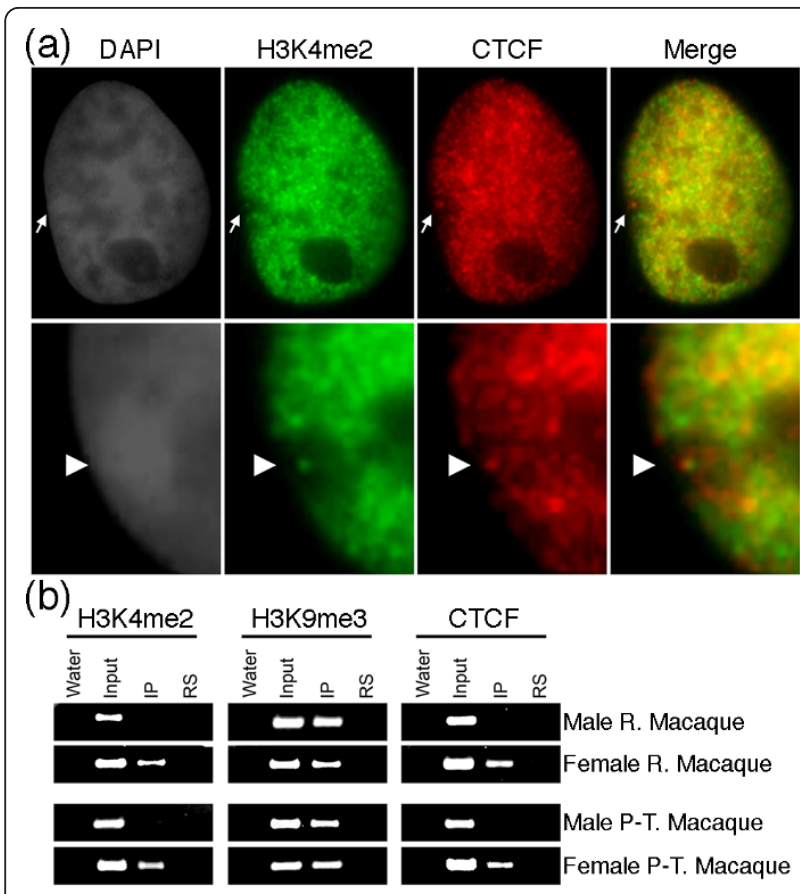

Figure 7 Female-specific association of CTCF and H3K4me2 with the macaque Xi. (a) Co-localization of CTCF (red) at the H3K4me2 'dot' (green) in the territory of the $X i$ in a female rhesus macaque nucleus. The white arrow indicates the $\mathrm{Xi}$ (top panel). The lower panel shows an independent example zoomed in. The white arrowhead points to the territory of the Xi. Nuclei are counterstained with DAPI, and the collected image converted to gray-scale to emphasize the Barr body. (b) Chromatin immunoprecipitation assessing the association of $\mathrm{H} 3 \mathrm{~K} 4 \mathrm{me2}$ H3K9me3 and CTCF with DXZ4 in male and female rhesus macaque (R. Macaque) and pig-tailed macaque (P-T. Macaque) chromatin. Samples include a no template control (Water), input DNA (Input), immunoprecipitation with the antibody indicated (IP) and nonspecific rabbit serum (RS).

Our data indicate that DXZ4 in the great apes and in the Old and New World monkeys is a polymorphic tandem-repeat, with array sizes comparable to those observed in humans [3]. DNA sequence data obtained from a BAC clone also provides evidence of tandem arrangement for the orthologous array in ring-tailed lemurs.

DNA sequence analysis reveals 95 to $97 \%$ sequence identity to human DXZ4 in the great apes, $90 \%$ in the lesser apes, $87 \%$ in Old World monkeys and $77 \%$ in New World monkeys. All of these primates had a repeat unit size around $3 \mathrm{~kb}$, high GC content (61 to 65\%) and a high incidence of CpG (154 to 192 per monomer). Human DXZ4 contains three internal microsatellite repeats that are the only repeat masked portion of a monomer. Through Old World monkeys the [GGGCC] and $[\mathrm{CT}]$ repeats are conserved. The [TAAA] repeat sequence is only conserved in chimpanzee and diverges in the other primates. However, all of the other primates have a simple repeat sequence that is enriched in $\mathrm{A}$ nucleotides at this location in the monomer, suggesting that retention of A-rich repetitive DNA is important for this region. In the common marmoset (a New World monkey), all three repeat sequences have diverged, but remain repetitive and retain $\mathrm{G}$-rich, $\mathrm{C}$-rich and A-rich sequence composition, respectively. It is generally accepted that DNA sequence composition influences nucleosome positioning [40]. Close examination of predicted nucleosome occupancy [41] for human DXZ4 using the UCSC genome browser [42] shows that the [TAAA] and [CT] repeats are strongly inhibitory of nucleosome occupancy, whereas the [GGGCC] repeat sequence resides at a peak of nucleosome occupancy (data not shown), suggesting that these sequences influence the position of nucleosomes in the array. Indeed, microarray hybridization using DNA isolated from human chromatin immunoprecipitated with antibodies to H3K4me2 and H3K9me3 revealed well defined peaks of modified nucleosomes predicted to be approximately every fourth nucleosome in DXZ4 [12], suggesting that nucleosome distribution within the array is likely well defined. Therefore, it is tempting to suggest that retention of base composition and location of these repeat sequences in primate DXZ4 is necessary to assist in maintaining nucleosome distribution and chromatin structure within the array.

Conservation of DXZ4 DNA sequence drops off rapidly in the lemurs, galago and tarsier branches of the primate tree. However, sequence homology extends across an approximately 400-bp region of DXZ4 encompassing the bidirectional promoter and binding sites for TFIID and CTCF [12]. Unlike the chromosome 4 macrosatellites RS447 [4] and D4Z4 [6], which both contain ORFs that are conserved through rodents [6], DXZ4 does not obviously encode a protein. Therefore, retention of this sequence is very significant, and suggestive of an important role for DXZ4 in primates as a genomic element, involving CTCF binding and transcription. Preliminary analysis of this region throughout mammals (including mouse) indicates the presence of a tandem repeat downstream of PLS3 that shows some homology to this 400-bp sequence, further supporting an important role for this element on the X chromosome (BP Chadwick, unpublished data), and this is now a major focus for our DXZ4 investigation.

Despite a lack of conserved ORFs, DXZ4 is expressed in humans. Most DXZ4 RNA are sense transcripts originating from the $\mathrm{Xa}$, although detectable anti-sense transcription is found specifically in females and therefore likely originates from the $\mathrm{Xi}$ [12]. Here we find that DXZ4 is expressed in male and female macaque, although almost all transcription appears to originate form the Xa and no anti-sense transcript was detected. 


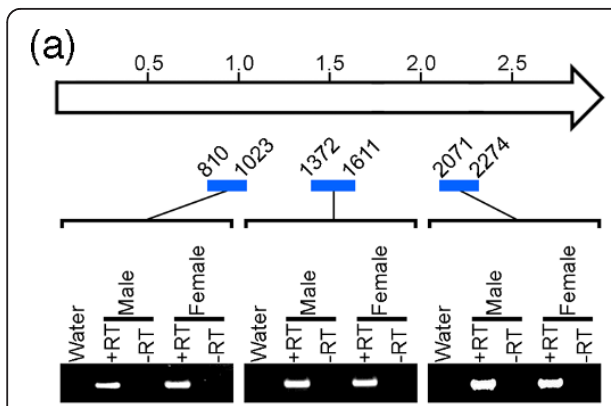

(b)

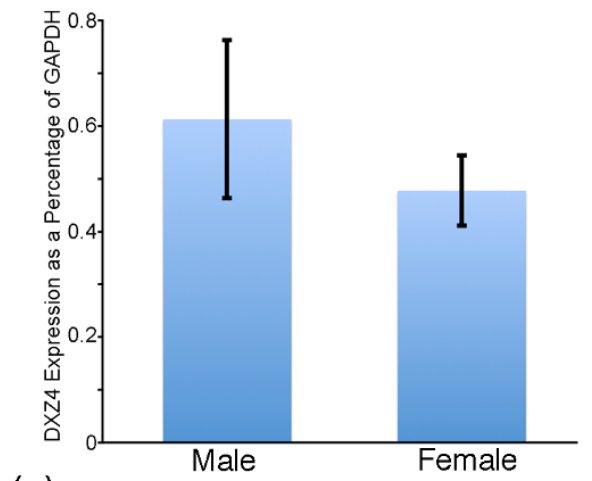

(c)
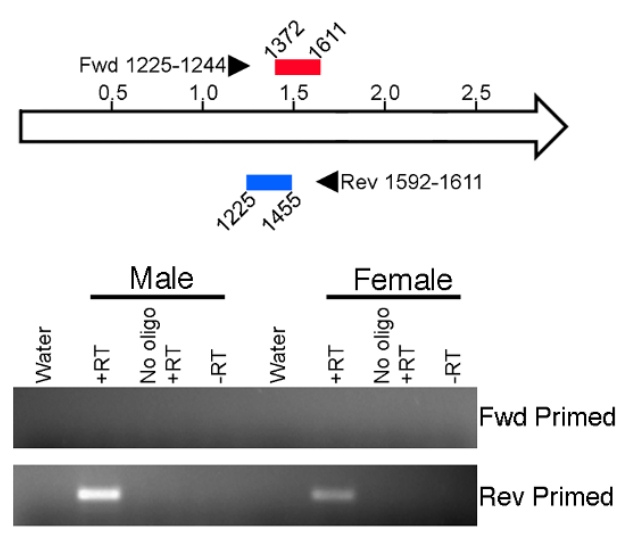

(e)
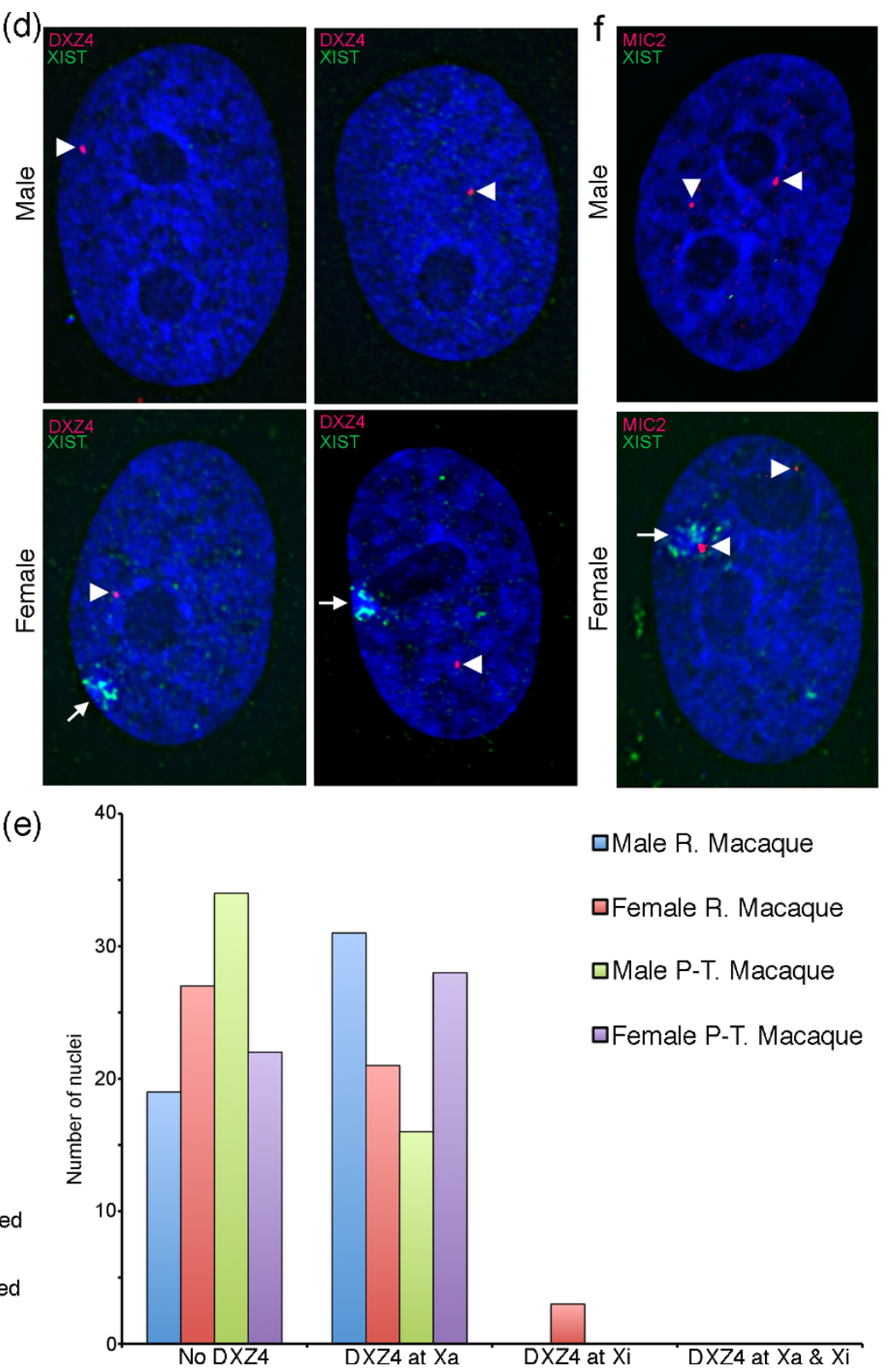

Figure 8 Expression of DXZ4 in male and female macaques. (a) Schematic representation of a single DXZ4 monomer with positions in kilobases indicated. Below the monomer the blue bars indicate the relative positions of regions of macaque DXZ4 assessed by RT-PCR with the coordinates of the fragment indicated. Each blue bar points to the corresponding RT-PCR data obtained from male and female rhesus macaque CDNA. Samples indicate a no template control (Water) and CDNA prepared form total RNA with (+RT) and without (-RT) including reverse

transcriptase enzyme. (b) Quantitative RT-PCR (qRT-PCR) analysis of DXZ4 expression in male and female rhesus macaque cDNA. Data represent the mean of four independent triplicate qRT-PCR analyses and the black bar indicates the standard error. Expression levels are represented as a percentage of GAPDH levels. (c) Strand-specific RT-PCR of DXZ4. The locations of primers used to prime anti-sense or sense cDNA synthesis are indicated by the right and left-facing arrow-heads and the precise location in DXZ4 is given by the coordinates. The red box represents the region assessed by PCR for anti-sense transcripts and the blue box for sense transcripts. Below the schematic map is the RT-PCR data as an ethidium bromide stained agarose gel. Samples include a no template control (Water), strand-specific primer with reverse transcriptase (+RT), no strand-specific primer with reverse transcriptase (No oligo +RT) and no primer with no reverse transcriptase (-RT). (d) RNA FISH analysis of DXZ4 expression in male and female rhesus macaque nuclei. Nuclei are counterstained with DAPI (blue). DXZ4 expression (red) is indicated by the white arrowheads. XIST RNA expression (green) is indicated by the white arrows. (e) Quantitative analysis of DXZ4 expression from the Xa and Xi in male and female rhesus macaque (R. Macaque) and pig-tailed macaque (P-T. Macaque) $(n=50)$. (f) RNA FISH analysis of MIC2 expression (red) relative to XIST (green) in male and female rhesus macaque nuclei. 
Therefore, expression of DXZ4 is conserved, but the significance of anti-sense transcription remains unclear.

In humans, DXZ4 in males and on the Xa in females is packaged into constitutive heterochromatin characterized by hyper-CpG methylation [3,12], H3K9me3 [12] and HP1 $\gamma$ [32]. Conversely, DXZ4 on the Xi is packaged, at least in part, into euchromatin characterized by $\mathrm{H} 3 \mathrm{~K} 4 \mathrm{me} 2$, H3K36me2, and histone acetylation, and is hypomethylated at $\mathrm{CpG}$ residues and bound by CTCF $[3,12,28,29]$. Both forms are expressed despite the contrasting chromatin packaging [12]. Here we show that all of these features are conserved at DXZ4 in the Old World monkey macaque. Furthermore, as has been observed in humans $[28,30,31]$, the macaque $\mathrm{Xi}$ is characterized by distinct reproducible bands of $\mathrm{H} 3 \mathrm{~K} 27 \mathrm{me} 3$, with the euchromatic form of DXZ4 located at the distal edge of a major Xq H3K27me3 band. Also consistent with the human Xi [28], additional distinct H3K4me2 signals reside at the distal edge of other $\mathrm{H} 3 \mathrm{~K} 27 \mathrm{me} 3$ bands, suggesting that conservation of this arrangement has some role in $\mathrm{Xi}$ chromatin organization. Therefore, determining the DNA sequence identity of these chromatin elements is a priority.

\section{Conclusions}

These data indicate several conserved features of DXZ4 that are likely important for the organization and function of the array: repeat monomer tandem arrangement; retention of high GC content and CpG incidence; conservation of the internal promoter sequence; conservation of the CTCF binding site; conservation of internal simple repeats; and array expression. Collectively, these features likely contribute to the roles of DXZ4 packaged into constitutive heterochromatin on the $\mathrm{Xa}$ and euchromatin bound by CTCF on the Xi. What function DXZ4 has in these contexts remains unclear. However, data from this study highlight important conserved features of DXZ4 that will assist in guiding the formulation of new hypotheses that can be tested to decipher the role of DXZ4 on the X chromosome. Furthermore, elucidation of DXZ4 function on the $\mathrm{Xi}$ will likely reveal additional intriguing parallels between the biology of DXZ4 and the contracted form of D4Z4 in FSHD, promoting our appreciation for these enigmatic genomic features.

\section{Materials and methods Cell culture}

Human female telomerase immortalized retinal pigment epithelial cells (hTERT-RPE1) were obtained from Clontech (Mountain View, CA, USA). Human male lymphoblastoid cell line GM06992 was obtained from the Coriell Cell Repositories (Coriell Institute for Medical Research), as were the following primate primary fibroblast cells: rhesus macaque (M. mulatta) AG08305 (male), and AG08312 (female); pig-tailed macaque ( $M$. nemistrina) AG07921 (male), and AG08452 (female); common squirrel monkey (Saimiri sciureus) AG05311 (female); black-handed spider monkey (Ateles geoffroyi) AG05352 (male). Cells were maintained according to Coriell's recommendations. Female gorilla (Gorilla gorilla) lymphoblast cells [43] were a gift from H Willard. Culture media (RPMI for lymphoblasts, and DMEM for fibroblasts), fetal bovine serum and supplements were all obtained from Invitrogen Corp (Carlsbad, CA, USA).

\section{Metaphase chromosome preparation}

In order to enrich for cells in metaphase, growth media of rhesus macaque primary fibroblasts was supplemented with colcemid (Invitrogen) to $25 \mathrm{ng} / \mathrm{ml}$ before returning cells to the incubator for an additional hour. Cells were harvested and resuspended in $37^{\circ} \mathrm{C} 75 \mathrm{mM} \mathrm{KCl}$ for 15 minutes. To this, one-sixth volume of fixative (three parts methanol to one part acetic acid) was applied before pelleting the cells. Cells were washed an additional six times with fixative, pelleting cells between each wash. Fixed cells were dropped from approximately $30 \mathrm{~cm}$ onto cleaned microscope slides resting on damp paper towels on top of a $37^{\circ} \mathrm{C}$ heat block. Slides were dried at room temperature for an additional 24 hours before use.

\section{Pulsed field gel electrophoresis, Southern blotting and hybridization}

Agarose embedded genomic DNA from primate cells were prepared essentially as described [5].

Agarose embedded DNA was digested with $\mathrm{XbaI}$ (NEB, Ipswich, MA, USA). Each plug was first equilibrated in $300 \mu \mathrm{l}$ of $1 \times$ digest buffer at room temperature for 20 minutes, before replacement of buffer with $100 \mu \mathrm{l}$ of $1 \times$ digest buffer containing 200 units of restriction enzyme. Digests were performed overnight at $37^{\circ} \mathrm{C}$. Plugs were loaded onto a $1.0 \%$ agarose gel prepared using pulsed field certified agarose (Biorad, Hercules, CA, USA) in $0.5 \times$ TBE. DNA was separated at $13^{\circ} \mathrm{C}$ in $0.5 \times$ TBE and conditions selected to separate 100 to $400 \mathrm{~kb}$ using the auto algorithm function of the CHEF Mapper (Biorad). Markers were loaded in the outer lanes (NEB, MidRange PFG Markers I and II). The gel was then rinsed with water before staining with ethidium bromide $(1 \mu \mathrm{g} / \mathrm{ml})$ at room temperature for 30 minutes. The gel was washed twice with water for 15 minutes each and an image captured. The gel was then treated with $0.25 \mathrm{M} \mathrm{HCl}$ for 15 minutes before denaturing for 30 minutes (1.5 M NaCl, 0.5 M NaOH). DNA was transferred to Hybond-N+ (GE Healthcare, Piscataway, NJ, USA) overnight by standard Southern blotting [44]. The membrane was rinsed with $2 \times$ SSC before baking at $120^{\circ} \mathrm{C}$ for 30 minutes. 
A DXZ4 probe was prepared by PCR amplification of regions of human DXZ4 with the following oligonucleotides: CAGGCAGAAATGAGCACCAC and TGGTGG CGGCCATGATCTG (485 bp); ACCAGGCAAACTG CCCAAG and TTCTGGTTTGTCAGGAAGGC (550 bp); ACCCTGTCCTTGGCAGATG and GTTGGACGTAGGCCAGGTG (491 bp); GCCTACGTCACGCAGGAAG and CCAGCGGAAAGTCCATGGG (402 bp); CACTTGGGAGACTCCTGAAC and TGTCCCCG AGGTTGTCTTG (485 bp); TCTCTCGCCCACTTCTACTG and GAGTCGATGGGCCTCTTAG (530 bp). The PCR products were cleaned (Qiagen, Valencia, CA, USA) before labeling with DIG-11-dUTP by random priming (Roche, Basel, Switzerland). The probes were tested for specificity and detection of the anticipated DNA fragment size on a Southern blot of EcoRI digested total genomic DNA.

Hybridization was performed overnight at $60^{\circ} \mathrm{C}$ using Expresshyb (Clontech). Blots were washed the following day at $60^{\circ} \mathrm{C}$ using two 8 -minute washes in $2 \times \mathrm{SSC}$, $0.1 \%$ SDS followed by one wash of 8 minutes in $0.2 \times$ SSC, $0.1 \%$ SDS. The probe was detected using anti-DIGalkaline phosphatase, blocking, wash and detection buffers according to the manufacturer's instructions (Roche). Signals were detected by exposure to photographic film (Kodak).

\section{BAC clone analysis}

Human BAC clone 2272M5 was obtained from Invitrogen. Human BAC clone RP11-268A15 was obtained from the Children's Hospital and Research Center at Oakland (CHRCO), as were the following green monkey (C. aethiops) and macaque BAC clones: macaque $(M$. mulatta) BAC clones from the CHORI-250 library CH250-131A6 and CH250-345N15; green monkey BAC clones from the CHORI-252 library - CH252-257K14, CH252-445A22, CH252-338G16 and CH252-199I1. Individual DXZ4 monomers from BAC clones $\mathrm{CH} 250$ 131A6 and CH252-338G16 were generated by first performing a HindIII digestion on the BAC clone DNA, gel purifying the $3-\mathrm{kb}$ fragment and cloning into calf intestinal alkaline phosphatase (NEB) treated HindIII cut pBluescript-II (Agilent Technologies, Santa Clara, CA, USA). Inserts were sequenced on both strands using $\mathrm{T} 7$, MM-F1 CCTCTTGATGGCAGTATTGC, MM-F2 C CTGGCCAGCATAGGTCAG, MM-F3 AGAGGCGG CAAGAGAAATGC, SP6, MM-R1 TTGTCAGGAAGGCAGGCTAG, MM-R2 ACATCGGGTTTCCGTCACAG and MM-R3 ATCCAACTTCCACCTCAACG.

\section{DNA and RNA FISH}

For DNA FISH, probes of human BAC clones RP11268A15 and 2272M5 were labeled with Spectrum Orange or Spectrum Green by nick translation according to the manufacturer's instructions (Abbott Molecular, Abbott Park, IL, USA), followed by ethanol precipitation in the presence of $25 \mu \mathrm{g}$ of human Cot-1 DNA (Invitrogen) and resuspension in $0.1 \mathrm{ml}$ of Hybrisol VII (MP Biomedicals, Solon, OH, USA). A 1:1 mix of the two probes was denatured at $75^{\circ} \mathrm{C}$ for 4 minutes, and repetitive sequences blocked at $37^{\circ} \mathrm{C}$ for 30 minutes before being applied directly to the slide, covered with cover glass, sealed with rubber cement and hybridized for 16 hours at $37^{\circ} \mathrm{C}$. Slides were washed at $37^{\circ} \mathrm{C}$ twice in $50 \%$ formamide, $2 \times \mathrm{SSC}$ for 8 minutes each, then once in $2 \times$ SSC for 8 minutes before adding ProLong Gold antifade containing DAPI (Invitrogen).

For RNA FISH, a direct-labeled Spectrum Green probe of human XIST exon 1 was prepared as described above and used with a Spectrum Red rhesus macaque 131A6 DXZ4 3-kb subclone probe. Fibroblasts were grown directly on slides before fixing and extracting in $4 \%$ formaldehyde, $0.1 \%$ Triton-X100 $1 \times$ phosphate buffered saline for 10 minutes at room temperature. Slides were washed for 2 minutes each in $1 \times$ phosphate buffered saline before dehydration through $70 \%$ and $100 \%$ ethanol for 2 minutes each and then air-drying. A 1:1 mix of the BAC and XIST probes was denatured at $72^{\circ} \mathrm{C}$ for 5 minutes before placing at $37^{\circ} \mathrm{C}$ for 30 to 60 minutes to block repetitive elements. The probe was applied onto cells and sealed with a coverslip and rubber cement at $37^{\circ} \mathrm{C}$ for 16 hours in a humidified chamber. Slides were washed twice at room temperature in $50 \%$ formamide, $2 \times$ sodium citrate sodium chloride (SSC), followed by 3 minutes at $37^{\circ} \mathrm{C}$ in $50 \%$ formamide $2 \times \mathrm{SSC}$ and one wash of 3 minutes at $37^{\circ} \mathrm{C}$ in $2 \times \mathrm{SSC}$ before addition of ProLong Gold antifade containing DAPI (Invitrogen). Control RNA FISH used a Spectrum Red MIC2 BAC clone RP11115101 from Invitrogen.

\section{Bisulfite sequencing}

Macaque genomic DNA was isolated from rheusus macaque and pig-tailed macaque cells using the Blood and Cell Culture DNA Midi-Kit (Qiagen), and bisulfite modified DNA prepared using the EpiTect Bisulfite kit (Qiagen) according to the manufacturer's recommendations. A 621-bp fragment of DNA was PCR amplified from bisulfite-modified DNA using the following primer pair: forward, GGGTATTAGGTAAATTGTTTA; reverse, CCATCCCAAAAACATAATTAAAA. PCR products were TA cloned into pDrive (Qiagen) and individual clones sequenced with M13R.

Immunofluorescence on interphase cells and metaphase chromosomes

Rabbit polyclonal anti-H3K4me2 (07-030), antiH3K4me3 (05-745), anti-CTCF (07-729), anti-H3K36me2 
(07-274) and anti-acetyl-lysine (06-933) were all obtained from Millipore (Billerica, MA, USA). Mouse monoclonal anti-H3K27me3 (ab6002) was obtained from Abcam (Cambridge, MA, USA). Secondary antibodies were obtained from Jackson ImmunoResearch Laboratories Inc. and Invitrogen (West Grove, PA, USA). Cell staining and preparation of metaphase chromosomes was performed essentially as described [28]. Images were collected using a Zeiss Axiovert 200M fitted with an AxioCam MRm and images managed using AxioVision 4.4 software (Carl Zeiss Microimaging, Inc.).

\section{Chromatin immunoprecipitation}

Chromatin immunoprecipitation using rhesus macaque and pig-tailed macaque cells was performed essentially as described [12]. Antibodies were obtained from Millipore: anti-CTCF (07-729), anti-H3K4me2 (07-030) and anti-H3K9me3 (07-523). Rabbit serum negative control was obtained from EMD (Gibbstown, NJ, USA). Immunoprecipitated DNA was PCR amplified using MM-F1 and MM-R1 (sequences given above).

\section{RT-PCR, strand-specific RT-PCR and quantitative RT-PCR}

Macaque total RNA was isolated from cells using the RNeasy Mini Kit (Qiagen). Residual genomic DNA was removed by pre-treating the RNA with DNaseI (Invitrogen) for 20 minutes at room temperature, before heat inactivating the DNaseI at $70^{\circ} \mathrm{C}$ in the presence of $2.5 \mathrm{mM}$ EDTA for 15 minutes. cDNA was prepared using $1 \mu \mathrm{g}$ of total RNA with or without M-MLV reverse transcriptase (Invitrogen) according to the manufacturer's instructions.

cDNA was amplified using Taq polymerase (NEB) with the following cycle: $95^{\circ} \mathrm{C}$ for 2 minutes, followed by 35 cycles of $95^{\circ} \mathrm{C}$ for 20 seconds, $58^{\circ} \mathrm{C}$ for $20 \mathrm{sec}-$ onds, $72^{\circ} \mathrm{C}$ for 30 seconds. Amplification used the following primers: MM-F1 and MM-R1; MM-F2 and MMR2; MM-F3 and MM-R3 (sequences given above).

Strand-specific cDNA was prepared essentially as described above except random hexamers were replaced with a strand-specific primer and an additional control was included of reverse transcriptase without primer. Anti-sense cDNA was primed with MM-F4 (TGACCAAGAGGTCAAAGGCG), whereas sense-strand cDNA was primed with MM-R2. cDNA was assessed with MM-F2 and MM-R2 or MM-F4 and MM-R4 (GTTGGACGTAGGCCAGGTG).

qRT-PCR was performed in triplicate four independent times using random primed cDNA with MM-F2 and MM-R4 using DyNAmo SYBR Green qPCR (NEB) on a CFX96 (Biorad).

\section{Abbreviations}

BAC: bacterial artificial chromosome; bp: base pair; CTCF: CCCTC binding factor; DAPI: 4',6-diamino-2-phenylinole dihydrochloride; FISH: fluorescence in situ hybridization; FSHD: facioscapulohumeral muscular dystrophy; H3K4me2: histone $\mathrm{H3}$ dimethylated at lysine 4; $\mathrm{H} 3 \mathrm{~K} 4 \mathrm{me} 3$ : histone $\mathrm{H3}$ trimethylated at lysine 4; H3K9me3: histone $\mathrm{H3}$ trimethylated at lysine 9; H3K27me3: histone $\mathrm{H} 3$ trimethylated at lysine $27 ; \mathrm{H} 3 \mathrm{~K} 36 \mathrm{me}$ : histone $\mathrm{H3}$ dimethylated at lysine 36; HP1Y: heterochromatin protein 1 gamma; kb: kilobase; ORF: open reading frame; qRT-PCR: quantitative reverse transcription polymerase chain reaction; RT-PCR: reverse transcription polymerase chain reaction; SSC: sodium citrate sodium chloride; VNTR: variable number tandem repeat; $X a$ : active $X$ chromosome; $X$ i: inactive $X$ chromosome; XIST: $X$ inactive specific transcript.

\section{Acknowledgements}

This work was supported by a grant from the National Institute of General Medical Sciences to BPC (NIH R01 GM073120). We are grateful to Hunt Willard for use of the gorilla cell line. We are also grateful to Rani Dhanarajan for assistance with subcloning green monkey and rhesus macaque DXZ4 monomers, and thank Jonathan Dennis for his helpful comments and discussion.

\section{Authors' contributions}

BPC conceived of the study, analyzed and interpreted data, performed experiments, and wrote the manuscript. CM carried out experiments and analyzed data.

\section{Competing interests}

The authors declare that they have no competing interests.

Received: 27 January 2011 Revised: 28 February 2011

Accepted: 13 April 2011 Published: 13 April 2011

\section{References}

1. Warburton PE, Hasson D, Guillem F, Lescale C, Jin X, Abrusan G: Analysis of the largest tandemly repeated DNA families in the human genome. BMC Genomics 2008 , 9.533.

2. Bruce HA, Sachs N, Rudnicki DD, Lin SG, Willour VL, Cowell JK, Conroy J, McQuaid DE, Rossi M, Gaile DP, Nowak NJ, Holmes SE, Sklar P, Ross CA, Delisi LE, Margolis RL: Long tandem repeats as a form of genomic copy number variation: structure and length polymorphism of a chromosome $5 p$ repeat in control and schizophrenia populations. Psychiatr Genet 2009, 19:64-71.

3. Giacalone J, Friedes J, Francke U: A novel GC-rich human macrosatellite VNTR in Xq24 is differentially methylated on active and inactive $\mathrm{X}$ chromosomes. Nat Genet 1992, 1:137-143.

4. Kogi M, Fukushige S, Lefevre C, Hadano S, Ikeda JE: A novel tandem repeat sequence located on human chromosome $4 \mathrm{p}$ : isolation and characterization. Genomics 1997, 42:278-283.

5. Tremblay DC, Alexander G Jr, Moseley S, Chadwick BP: Expression, tandem repeat copy number variation and stability of four macrosatellite arrays in the human genome. BMC Genomics 2010, 11:632.

6. van Deutekom JC, Wijmenga C, van Tienhoven EA, Gruter AM, Hewitt JE, Padberg GW, van Ommen GJ, Hofker MH, Frants RR: FSHD associated DNA rearrangements are due to deletions of integral copies of a $3.2 \mathrm{~kb}$ tandemly repeated unit. Hum Mol Genet 1993, 2:2037-2042.

7. Bakker E, Wijmenga C, Vossen RH, Padberg GW, Hewitt J, van der Wielen M, Rasmussen K, Frants RR: The FSHD-linked locus D4F104S1 (p13E-11) on 4q35 has a homologue on 10qter. Muscle Nerve 1995, 2:539-44.

8. Deidda G, Cacurri S, Grisanti P, Vigneti E, Piazzo N, Felicetti L: Physical mapping evidence for a duplicated region on chromosome 10qter showing high homology with the facioscapulohumeral muscular dystrophy locus on chromosome 4qter. Eur J Hum Genet 1995, 3:155-167.

9. Wijmenga C, Hewitt JE, Sandkuijl LA, Clark LN, Wright TJ, Dauwerse HG, Gruter AM, Hofker MH, Moerer P, Williamson R, et al: Chromosome 4q DNA rearrangements associated with facioscapulohumeral muscular dystrophy. Nat Genet 1992, 2:26-30

10. Tawil R: Facioscapulohumeral muscular dystrophy. Neurotherapeutics 2008 5:601-606

11. Lemmers RJ, Wohlgemuth $M$, van der Gaag KJ, van der Vliet PJ, van Teijlingen CM, de Knijff P, Padberg GW, Frants RR, van der Maarel SM: Specific sequence variations within the $4 q 35$ region are associated with facioscapulohumeral muscular dystrophy. Am J Hum Genet 2007, 81:884-894. 
12. Chadwick BP: DXZ4 chromatin adopts an opposing conformation to that of the surrounding chromosome and acquires a novel inactive X-specific role involving CTCF and antisense transcripts. Genome Res 2008 18:1259-1269.

13. Saitoh Y, Miyamoto N, Okada T, Gondo Y, Showguchi-Miyata J, Hadano S, Ikeda JE: The RS447 human megasatellite tandem repetitive sequence encodes a novel deubiquitinating enzyme with a functional promoter. Genomics 2000, 67:291-300.

14. Snider L, Asawachaicharn A, Tyler AE, Geng LN, Petek LM, Maves L, Miller DG, Lemmers RJ, Winokur ST, Tawil R, van der Maarel SM, Filippova GN, Tapscott SJ: RNA transcripts, miRNA-sized fragments, and proteins produced from D4Z4 units: new candidates for the pathophysiology of facioscapulohumeral dystrophy. Hum Mol Genet 2009, 18:2414-2430.

15. Hewitt JE, Lyle R, Clark LN, Valleley EM, Wright TJ, Wijmenga C, van Deutekom JC, Francis F, Sharpe PT, Hofker M, et al: Analysis of the tandem repeat locus D4Z4 associated with facioscapulohumeral muscular dystrophy. Hum Mol Genet 1994, 3:1287-1295.

16. Gabriels J, Beckers MC, Ding H, De Vriese A, Plaisance S, van der Maarel SM, Padberg GW, Frants RR, Hewitt JE, Collen D, Belayew A: Nucleotide sequence of the partially deleted D4Z4 locus in a patient with FSHD identifies a putative gene within each $3.3 \mathrm{~kb}$ element. Gene 1999, 236:25-32.

17. Dixit M, Ansseau E, Tassin A, Winokur S, Shi R, Qian H, Sauvage S, Mattéotti C, van Acker AM, Leo O, Figlewicz D, Barro M, LaoudjChenivesse D, Belayew A, Coppée F, Chen YW: DUX4, a candidate gene of facioscapulohumeral muscular dystrophy, encodes a transcriptional activator of PITX1. Proc Natl Acad Sci USA 2007, 104:18157-18162.

18. Lemmers RJ, van der Vliet PJ, Klooster R, Sacconi S, Camaño P, Dauwerse JG, Snider L, Straasheijm KR, van Ommen GJ, Padberg GW, Miller DG, Tapscott SJ, Tawil R, Frants RR, van der Maarel SM: A unifying genetic model for facioscapulohumeral muscular dystrophy. Science 2010, 329:1650-1653

19. Clapp J, Mitchell LM, Bolland DJ, Fantes J, Corcoran AE, Scotting PJ, Armour JA, Hewitt JE: Evolutionary conservation of a coding function for D4Z4, the tandem DNA repeat mutated in facioscapulohumeral muscular dystrophy. Am J Hum Genet 2007, 81:264-279.

20. Payer B, Lee JT: $X$ chromosome dosage compensation: how mammals keep the balance. Annu Rev Genet 2008, 42:733-772.

21. Mohandas T, Sparkes RS, Shapiro LJ: Reactivation of an inactive human $X$ chromosome: evidence for $\mathrm{X}$ inactivation by DNA methylation. Science 1981, 211:393-396.

22. Pfeifer GP, Tanguay RL, Steigerwald SD, Riggs AD: In vivo footprint and methylation analysis by PCR-aided genomic sequencing: comparison of active and inactive $\mathrm{X}$ chromosomal DNA at the CpG island and promoter of human PGK-1. Genes Dev 1990, 4:1277-1287.

23. Boggs $B A$, Cheung $P$, Heard E, Spector DL, Chinault AC, Allis CD: Differentially methylated forms of histone $\mathrm{H} 3$ show unique association patterns with inactive human X chromosomes. Nat Genet 2002, 30:73-76.

24. Peters AH, Mermoud JE, O'Carroll D, Pagani M, Schweizer D, Brockdorff N, Jenuwein $\mathrm{T}$ : Histone $\mathrm{H} 3$ lysine 9 methylation is an epigenetic imprint of facultative heterochromatin. Nat Genet 2002, 30:77-80

25. Plath K, Fang J, Mlynarczyk-Evans SK, Cao R, Worringer KA, Wang H, de la Cruz CC, Otte AP, Panning B, Zhang Y: Role of histone H3 lysine 27 methylation in X inactivation. Science 2003, 300:131-135.

26. Silva J, Mak W, Zvetkova I, Appanah R, Nesterova TB, Webster Z, Peters AH, Jenuwein T, Otte AP, Brockdorff N: Establishment of histone h3 methylation on the inactive $x$ chromosome requires transient recruitment of eed-enx1 polycomb group complexes. Dev Cell 2003, 4:481-495.

27. Jeppesen $P$, Turner $B M$ : The inactive $X$ chromosome in female mammals is distinguished by a lack of histone $\mathrm{H} 4$ acetylation, a cytogenetic marker for gene expression. Cell 1993, 74:281-289.

28. Chadwick BP, Willard HF: Cell cycle-dependent localization of macroH2A in chromatin of the inactive X chromosome. J Cell Biol 2002, 157:1113-1123.

29. Chadwick BP, Willard HF: Chromatin of the Barr body: histone and nonhistone proteins associated with or excluded from the inactive $X$ chromosome. Hum Mol Genet 2003, 12:2167-2178.

30. Chadwick BP: Variation in Xi chromatin organization and correlation of the H3K27me3 chromatin territories to transcribed sequences by microarray analysis. Chromosoma 2007, 116:147-157.
31. Chadwick BP, Willard HF: Multiple spatially distinct types of facultative heterochromatin on the human inactive $\mathrm{X}$ chromosome. Proc Natl Acad Sci USA 2004, 101:17450-17455.

32. Zeng W, de Greef JC, Chen YY, Chien R, Kong X, Gregson HC, Winokur ST, Pyle A, Robertson KD, Schmiesing JA, Kimonis VE, Balog J, Frants RR, Ball AR Jr, Lock LF, Donovan PJ, van der Maarel SM, Yokomori K: Specific loss of histone $\mathrm{H} 3$ lysine 9 trimethylation and HP1gamma/cohesin binding at D4Z4 repeats is associated with facioscapulohumeral dystrophy (FSHD). PLoS Genet 2009, 5:e1000559.

33. van Overveld PG, Lemmers RJ, Sandkuijl LA, Enthoven L, Winokur ST, Bakels F, Padberg GW, van Ommen GJ, Frants RR, van der Maarel SM: Hypomethylation of D4Z4 in 4q-linked and non-4q-linked facioscapulohumeral muscular dystrophy. Nat Genet 2003, 35:315-317.

34. Ottaviani A, Rival-Gervier S, Boussouar A, Foerster AM, Rondier D, Sacconi S, Desnuelle C, Gilson E, Magdinier F: The D4Z4 macrosatellite repeat acts as a CTCF and A-type lamins-dependent insulator in facio-scapulo-humeral dystrophy. PLoS Genet 2009, 5:e1000394.

35. Chadwick BP: Macrosatellite epigenetics: the two faces of DXZ4 and D4Z4. Chromosoma 2009, 118:675-681.

36. Samonte RV, Conte RA, Verma RS: Localization of human midisatellite and macrosatellite DNA sequences on chromosomes 1 and $X$ in the great apes. J Hum Genet 1999, 44:57-59.

37. ClustalW2 - Multiple Sequence Alignment.. [http://www.ebi.ac.uk/Tools/ $\mathrm{msa} /$ clustalw2/].

38. Rhesus Macaque Genome Sequencing and Analysis Consortium, Gibbs RA Rogers J, Katze MG, Bumgarner R, Weinstock GM, Mardis ER, Remington KA, Strausberg RL, Venter JC, Wilson RK, Batzer MA, Bustamante CD, Eichler EE, Hahn MW, Hardison RC, Makova KD, Miller W, Milosavljevic A, Palermo RE, Siepel A, Sikela JM, Attaway T, Bell S, Bernard KE, Buhay CJ,

Chandrabose MN, Dao M, Davis C, Delehaunty KD, et al: Evolutionary and biomedical insights from the rhesus macaque genome. Science 2007, 316:222-234

39. Xing J, Witherspoon DJ, Ray DA, Batzer MA, Jorde LB: Mobile DNA elements in primate and human evolution. Am J Phys Anthropol 2007, , Suppl 45: 2-19.

40. Segal E, Fondufe-Mittendorf $Y$, Chen $L$, Thastrom A, Field $Y$, Moore IK, Wang JP, Widom J: A genomic code for nucleosome positioning. Nature 2006, 442:772-778

41. Dennis JH, Fan HY, Reynolds SM, Yuan G, Meldrim JC, Richter DJ, Peterson DG, Rando OJ, Noble WS, Kingston RE: Independent and complementary methods for large-scale structural analysis of mammalian chromatin. Genome Res 2007, 17:928-939.

42. UCSC Genome Browser.. [http://genome.ucsc.edu].

43. Neubauer RH, Rabin H, Strnad BC, Nonoyama M, Nelson-Rees WA: Establishment of a lymphoblastoid cell line and isolation of an EpsteinBarr-related virus of gorilla origin. J Virol 1979, 31:845-848.

44. Sambrook J, Fritsch EF, Maniatis T: Molecular Cloning: A Laboratory Manual. 2 edition. Cold Spring Harbor, NY: Cold Spring Harbor Laboratory Press; 1989.

45. Edgar RC: MUSCLE: multiple sequence alignment with high accuracy and high throughput. Nucleic Acids Res 2004, 32:1792-1797.

doi:10.1186/gb-2011-12-4-r37

Cite this article as: McLaughlin and Chadwick: Characterization of DXZ4 conservation in primates implies important functional roles for CTCF binding, array expression and tandem repeat organization on the $\mathrm{X}$ chromosome. Genome Biology 2011 12:R37. 